\title{
IV.4 Das Projekt der trilateralen Nuklearkooperation 1956-1958
}

\section{IV.4.1 Sondierungen: Atomforschung und Luftfabrtindustrie 1950-1955}

\section{Kontrolle und Kommerz}

Wohl keines der zahlreichen Gebiete deutsch-französischer Zusammenarbeit belegt die mit der Ratifikation der Pariser Verträge 1955 und der Beseitigung des saarländischen Stolpersteins 1956 gewonnene neue Qualität der bilateralen Beziehungen so gut wie das 1957 voll einsetzende Tête-à-tête in der Nuklearrüstungspolitik ${ }^{1}$. Für Eingeweihte war es freilich keineswegs so sensationell, wie es dem außenstehenden Betrachter erscheinen mochte. Mit guten Gründen kann man daher die These aufstellen, daß die Rüstungskooperation Mitte der fünfziger Jahre „nicht neu einsetzt, sondern lediglich neu ansetzt“2. Erwägungen zu einer bilateralen Kernwaffenkooperation gab es letztlich seit Beginn der ersten innerfranzösischen Planungen über die Möglichkeiten einer atomaren Verteidigung. Schon 1950 hatten französische Militärs die Forderung nach einer französischen Atombombe aufgestellt ${ }^{3}$. Noch im selben Jahr unterbreitete Paris der Bonner Regierung das bemerkenswerte Angebot, sich an einer europäischen Forschungsstation für Atomenergie zu beteiligen ${ }^{4}$. Wenige Monate später, im Mai 1951, setzte Jean Monnets Commissariat Général du Plan das Kanzleramt von der Absicht in Kenntnis, ein bilaterales Institut für die reine und angewandte Forschung zur Integration der europäischen Industrie aufbauen zu wollen. Als Arbeitsfeld war an das Studium der Forschungs- und Investitionsprobleme mit strategischem Charakter im Rahmen der militärischen Verteidigung Europas gedacht ${ }^{5}$. Im deutschen Generalkonsulat in Paris wurde das Vorhaben durchaus begrüßt, sah man darin doch die Chance, „daß Deutschland auf diese Weise in die Atomforschung einbezogen werden könnte, wozu Anlagen und Laboratorien erforderlich sind, die über die finanzielle Leistungsfähigkeit eines einzelnen Landes hinausgehen "6.

Ende Mai kam deutschen Diplomaten an der Seine das Gerücht zu Ohren, im Rahmen eines von deutscher Seite entwickelten neuen Plans zur Koordinierung der europäischen Atomforschung sei die Serienfabrikation von Atomwaffen durch eine deutsch-französische Behörde mit Sitz in Tübingen vorgesehen. Außerdem solle die schon finanzierte französische Atombatterie P 2 in der Nähe von Düsseldorf gebaut werden?. Die vom aufgeschreckten Auswärtigen Amt sofort in die Wege geleiteten Recherchen ergaben, daß die deutschen Physiker eine solche

1 Einen umfassenden Überblick über die deutsch-französischen Beziehungen nach 1955 unter dem Blickwinkel der sicherheitspolitischen Komponente bieten: Kocs, Autonomy; Soutou, L'alliance incertaine.

2 Albrecht, Rüstungsfragen, S. 138.

3 Zur Entwicklung der militärischen französischen Nuklearpolitik vgl. Ailleret, Aventure atomique; Goldschmidt, Complexe atomique; ders., Rivalités; Rocard, La naissance; Scheinman, Atomic energy policy; Schmitt, Frankreich.

4 S. PA, Abt. 3, AZ 230-04, Hausenstein an DfAA, 230 Nr. 1346, 6. 12.1950.

5 S. ebd., NL Hausenstein, Bd. 29, Bl. 5, Sänger an Hausenstein, 9. 5. 1951; ebd., Bl. 10f., „De la recherche pure et appliqué à l'intégration de l'industrie européenne“, VG/JD/51/04/16, o.D.

6 Ebd., Bl. 12, von Kessel an AA, 460-03 Tgb.Nr. 1692, 12. 5. 1951, Konzept.

7 S. ebd, Abt. 3, AZ 230-04, Hausenstein an dass., 230-04 Tgb.Nr. 1959, 28. 5. 1951. 
Kooperation rundweg bestritten und wegen der aufzubringenden enormen finanziellen Mittel auch „zur Zeit keinerlei Interesse" besaßen, sich auf eine derartige Koordinierung im militärischen Bereich einzulassen ${ }^{8}$. Trotz dieses Dementis fragten sich die Beamten in der Koblenzer Straße, ob sich nicht doch jüngere deutsche Wissenschaftler an einem solchen Projekt beteiligten, und wandten sich umgehend an den Physiker Carl Friedrich von Weizsäcker'. Nach Rücksprache mit seinem Kollegen Werner Heisenberg wußte von Weizsäcker zu berichten, daß es tatsächlich offiziöse Kontakte gebe, wobei Heisenberg seine Gesprächspartner aber auf den offiziellen Weg verwiesen habe. Laut von Weizsäcker waren seine Kollegen an einer rein wissenschaftlichen Zusammenarbeit sehr interessiert, nahmen auch an gemeinsamen Arbeiten zur friedlichen Nutzung der Kernenergie teil, hegten aber "große Bedenken" gegen eine Mitarbeit an der Entwicklung von Atomwaffen ${ }^{10}$. Auf seine Anregung hin erkundigte sich das Auswärtige Amt auch noch bei den Landesregierungen von Württemberg-Baden und Nordrhein-Westfalen. Doch auch ihnen lagen über die in Paris kursierenden Vermutungen keine Informationen vor ${ }^{11}$.

Wenige Monate später, im September 1951, beschloß der in der französischen Regierung für nukleare Fragen zuständige Staatssekretär Félix Gaillard insgeheim, das "Commissariat à l'Energie Atomique“ (CEA) mit den vorbereitenden Arbeiten zum Bau einer Atombombe zu beauftragen und dafür die jährliche Produktion von 50 Kilogramm Plutonium vorzusehen ${ }^{12}$. Kurz darauf verständigten sich deutsche und französische Diplomaten im Rahmen der Verhandlungen über die Europäische Verteidigungsgemeinschaft darauf, den Gedanken einer Europäisierung der Rüstungsproduktion einzubringen und auch das Problem der Atomforschung zu erörtern ${ }^{13}$.

In den USA wurden diese Bestrebungen mißtrauisch verfolgt. Im Dezember empfahl der amerikanische Hohe Kommissar dem Kanzler ein freiwilliges, per Gesetz verbrieftes Verbot zur Produktion von ABC-Waffen, Raketen, Flugzeugen und bestimmten Marinerüstungen. McCloy sagte zugleich zu, daß die Alliierten ihre eigene Gesetzgebung entsprechend abänderten. Adenauer bestand aber darauf, die Bundesrepublik keinerlei Diskriminierung zu unterwerfen, und zeigte sich zuversichtlich, das Problem der Sicherheitskontrolle innerhalb der EVG lösen zu können. Nur wenn dieser Weg nicht möglich sei, wollte er McCloys Überlegung in Erwägung ziehen, sofern deutsche Wissenschaftler an nuklearen Forschungen im Ausland teilnehmen könnten und Bonn der Bau ziviler Flugzeuge erlaubt würde ${ }^{14}$. Fünf Monate später einigten sich die drei westlichen Außenmini-

8 Ebd., Feihl an von Etzdorf, Aufzeichnung, 15.6.1951.

9 S. ebd., ders, an von Weizsäcker, 230-04-IIIb 176/51, 19. 6. 1951, Konzept.

10 Ebd., von Weizsäcker an Feihl, 30. 6. 1951; zur distanzierten Position Heisenbergs gegenüber der militärischen Nutzung der Kernenergie vgl. Fischer, Atomenergie, S. $29 \mathrm{f}$.

11 S. PA, Abt. 3, AZ 230-04, Feihl an Vertretung von Württemberg-Baden beim Bund, 230-04 IIIb2474/51, 6. 7. 1951, Konzept; ebd., Amann an AA, Nr. 7811, 27. 7. 1951; ebd., Spiecker an dass., Tgb.Nr. 5113/51, 21.8. 1951.

12 S. Bariéty, Frankreich und das Scheitern der EVG, S. 106; Le Douarec, Félix Gaillard, S. 67; Scheinman, Atomic energy policy, S. $63 \mathrm{f}$.

13 S. PA, Abt. 2, Bd. 1018, Besprechung über die EVG-Konferenz vom 13. 10. 1951, Streng vertraulich.

14 S. McCloy an Acheson, Tel. 801, 19. 12. 1951, Secret, in: FRUS 1951, Bd. 3, S. 1739-1741. 
ster auf eine als Anhang zum Artikel 107 des EVG-Vertrags fixierte Verbotsliste deutscher Waffenfabrikationen ${ }^{15}$, die zusammen mit Adenauers „Brief über die Atomenergie“ vom 7. 5. $1952^{16}$ die Verpflichtung der Bundesrepublik zu weitreichenden Kontrollen bzw. Einschränkungen bei der Rüstungsproduktion vertraglich fixierte ${ }^{17}$.

Frankreich ging jetzt in der Nuklearfrage seiner eigenen Wege und arbeitete unter der Federführung Gaillards einen Fünfjahresplan aus, der es dem CEA erlauben sollte, das Stadium der Laborversuche zu verlassen und mit Plutoniumreaktoren in die industrielle Produktion zu gehen ${ }^{18}$. Als Ersatzfeld deutsch-französischer Kooperation erschloß man nun den Flugzeugbau' ${ }^{19}$. Anfang September nahmen Vertreter des Bundes, des Landes Nordrhein-Westfalen und der Luftfahrtindustrie sowie der Berichterstatter für Luftverkehrsfragen im französischen Senat, Pellenc, Gespräche über die Möglichkeit einer Zusammenarbeit durch den Bau von Werken in Marokko auf ${ }^{20}$. Parallel dazu trat die französische Luftfahrtindustrie mit deutschen Technikern in Kontakt, die großes Interesse an einer Mitarbeit im Flugzeugbau anmeldeten ${ }^{21}$. Als das deutsch-französische Verhältnis aufgrund mannigfacher Probleme und Zwistigkeiten ${ }^{22}$ Ende Oktober einen "Tiefstand" erreichte, dachte Adenauer laut darüber nach, eine engere wirtschaftliche Kooperation „im Wege über eine Arbeitsteilung bei Rüstungsaufträgen“ anzubahnen ${ }^{23}$. Paris hielt sich aber bedeckt. Verteidigungsminister Pleven konnte sich diese Zusammenarbeit „,vorläufig nur auf dem Wege über die Aufträge für die Rüstungswirtschaft vorstellen“24. Außenminister Schuman ging noch einen Schritt weiter und brachte Ende November Pleven gegenüber sein starkes Unbehagen über die Kontakte der französischen Industrie mit deutschen Luftfahrtkonstrukteuren zum Ausdruck ${ }^{25}$. Zwar waren die im Pariser Vertrag enthaltenen Verbote bewußt so angelegt, daß sie „une coopération franco-allemande en dehors du territoire de l'Allemagne" nicht ausschlossen²6. Doch hegte der Quai d'Orsay große Sorge, die Entwicklung der deutschen Rüstungsindustrie doch nicht völlig unter Kontrolle halten zu können. Derartige Bedenken fand man in der Rue Saint-Dominique fehl am Platze. Da man sich zu diesem heiklen Thema nicht schriftlich äußern mochte, ließ man die Sache monatelang ruhen und lud dann Ende April

15 S. Art. 107 des EVG-Vertrages, 27. 5. 1952, in: BGBl 1954, Teil II, S. 371.

16 S. Adenauer an Acheson und Eden, 7. 5. 1952, in: ebd., S. 416; ders. an die Außenminister der EVG-Staaten, 7. 5. 1952, in: ebd., S. $416 \mathrm{f}$.

17 S. AMAE, Europe 1944-1960, Allemagne, Bd. 1050, Bl. 243-247, Konferenz der drei westlichen Außenminister vom 24. 5. 1952, Très secret; Treffen ders, vom 24. 5. 1952, Aufzeichnung, Secret, in: FRUS 1952-1954, Bd. 7, S. 92 f.; Bonner Konferenz vom 24. 5. 1952, in: AAPD, Adenauer und die Hohen Kommissare 1952, S. 344f.; Fischer, Atomenergie, S. 46-51.

18 S. Bariéty, Frankreich und das Scheitern der EVG, S. 108; Goldschmidt, Rivalités, S. 194 f.; Le Douarec, Félix Gaillard, S. 67 f.; Scheinman, Atomic energy policy, S. 74-89.

19 Vgl. auf der Basis amerikanischer Akten Abelshauser, Rüstung, S. $33 \mathrm{f}$.

20 S. PA, Abt. 3, AZ 752-00/22, von Etzdorf an Lahr, 331-14 III 1325/52, 12. 9. 1952.

21 S. AMAE, Europe 1944-1960, Allemagne, Bd. 498, B1. 87-90, "Projets de coopération entre l'industrie aéronautique allemande et les industries similiaires de France et de Grande-Brétagne“, 10. 10. 1952.

22 Vgl. Kap. III.1.3, III.2.2, III.3.2 u. III.4.2.

23 BA, NL Blankenhorn, Bd. 14b, Bl. 167, Tb. 29. 10. 1952

24 PA, BStS, Bd. 217, Hallstein an Adenauer, Aufzeichnung StS 2076/52, 17. 11. 1952.

25 S. AMAE, Europe 1944-1960, Allemagne, Bd. 498, Bl. 168-174, Schumann an Pleven, 27. 11. 1952.

26 Aufzeichnung Sauvagnargues, 10. 7. 1954, in: BDFD II, S. 218-221, hier S. 220. 
1953 Beamte aus dem Außenministerium zu einem Meinungsaustausch ein. Dabei wurde rasch deutlich, wie sehr die Positionen zwischen den beiden Ressorts differierten. Da die französische Luftfahrtindustrie seit $1944 \mathrm{zu}$ einem bedeutenden Teil von der Arbeit deutscher Ingenieure in Frankreich abhing und es nur mit ihrer Hilfe möglich war, sich der Konkurrenz zu widersetzen, setzten die Beamten im Verteidigungsministerium fest auf die Zusammenarbeit mit den Deutschen. Neben der Nutzbarmachung ihres technologischen Wissens ging es den Franzosen aber noch um etwas anderes ${ }^{27}$. Die NATO hatte im Dezember 1952 die auf der Lissabonner Ministerratstagung im Februar beschlossene Doppelstrategie von nuklearem Schild und konventionellem Schwert kodifiziert und damit der Bundesrepublik die Hoffnung eingeimpft, mit ihrer geplanten Gesamtstärke von ca. 498000 Mann ein „konventioneller Machtfaktor“ zu werden²8. Damit eröffnete sich Frankreich wiederum die Chance, die Bundesrepublik mit ihrem Rüstungsmaterial auszustatten. Da sich die Absatzbedingungen für die französischen Maschinen auf der anderen Rheinseite zusehends verschlechterten, glaubte das Verteidigungsministerium, die eigene Luftfahrtindustrie gegenüber der angelsächsischen und entstehenden deutschen Konkurrenz nur retten zu können, wenn man sich zu einer weiten Assoziation mit den Deutschen zusammenschlösse ${ }^{29}$.

Im Quai d'Orsay blieb man dennoch skeptisch, was sogar unter französischen Diplomaten auf Unverständnis stieß. Ganz im Sinne Plevens warnte FrançoisPoncet Frankreich vor der Gefahr, von den Angelsachsen völlig ausgebootet zu werden. Da die westlichen Partner offenbar nur wenig auf die im EVG-Vertrag festgelegten Restriktionen gaben, drohte die Bundesrepublik im Bereich der Luftfahrt zu ihrem Satelliten zu werden. Nur mit Hilfe einer „entente des industriels français et allemands“ glaubte er die französischen Interessen retten zu können. Noch bot sich dazu eine Gelegenheit. Ließe man sie ungenutzt verstreichen, so führte der Hohe Kommissar Anfang Dezember Außenminister Bidault vor Augen, nähme die Zukunft Frankreichs im industriellen wie militärischen Sektor schweren Schaden ${ }^{30}$.

Im Juli 1954 legte die französische Industrie in Absprache mit dem Luftfahrtministerium Pläne vor, die den Bau einer europäischen Luftfahrtfabrik in Nordafrika unter deutscher Beteiligung mit dem Ziel vorsahen, „de fabriquer des appareils de guerre, en prévision notamment d'une prochaine contribution allemande à la défense." Noch immer hatte der Quai d'Orsay seine Sorge nicht völlig abgelegt, daß die Deutschen mittels bilateraler Kooperation dazu in die Lage versetzt werden könnten, eine eigene Luftfahrtindustrie aufzubauen und die Beschränkungen des EVG-Vertrags zu unterlaufen. Allerdings stellten sich die Beamten nunmehr die Frage, ob man sich den Bemühungen der Industrie nicht doch besser anschlieBen, sie aber unter die Fittiche der Regierung nehmen sollte, um die alliierten Restriktionen im Rüstungsbereich gegenüber der Bundesrepublik zu konservieren ${ }^{31}$.

27 S. Aufzeichnung der Unterabt. Zentraleuropa im MAE, 5. 5. 1953, in: ebd., S. 190; zur Rekrutierung deutscher Rüstungsspezialisten durch Frankreich in den ersten Nachkriegsjahren vgl. Albrecht, Rüstungsfragen; Bossuat, Armements, S. 601-603.

28 Greiner, Zwischen Integration und Nation, S. 269.

29 S. Aufzeichnung der Unterabt. Zentraleuropa im MAE, 5. 5. 1953, in: BDFD II, S. 190-192.

30 S. François-Poncet an Bidault, Nr. 2468, 1. 12. 1953, in: ebd., S. 194 f., hier S. 194.

31 Abt. Europa im MAE an Mendès France, Aufzeichnung, ca. 5. 7. 1954, in: ebd., S. 216 f., hier S. 217. 
Unter dem Druck der Verhältnisse, d.h. der schweren EVG-Krise und der eigenen Nuklearambitionen im militärischen Bereich, öffnete sich das Ministerium jetzt in internen Planspielen einer Entente mit der Bundesrepublik auch auf den Sektoren der Atom- und Raketenwaffen. Das Motiv lag auf der Hand: Kontrolle des deutschen Rüstungspotentials und Anzapfung der technischen wie finanziellen Ressourcen. Die eigenen Kernwaffenplanungen waren inzwischen so weit gediehen, daß der Bau einer Bombe für Frankreich als machbar galt und die militärische Führung verlangte, die Streitkräfte müßten sich dezidiert zur Atomforschung hin orientieren ${ }^{32}$. Zusätzlichen Antrieb gewannen diese Forderungen durch den seit Herbst 1953 immer offener diskutierten „New Look“ in der amerikanischen Verteidigungskonzeption: Konzentrierung auf nukleare Waffensysteme bei gleichzeitiger Vernachlässigung konventioneller Verteidigung und Neigung zur Truppenreduzierung in Europa ${ }^{33}$. Mitte Dezember hatte der NATO-Ministerrat auf seiner Tagung in Paris die Umstellung der bisherigen Militärstrategie akzeptiert und sich somit darauf verständigt, einer Bedrohung auch mit nuklearen Waffen zu begegnen ${ }^{34}$. Frankreich reagierte auf diesen Strategiewandel hin zur "massive retaliation" mit der politischen Entscheidung für den Bau der Atomwaffe. Am 17.3. 1954 informierte Pleven das Parlament über die Forschungen des CEA im nuklearen Bereich. Zwei Tage später sprach er sich auch öffentlich für eine Kernwaffe aus und gab damit zu verstehen, daß die Regierung die Entscheidung zum Bau einer Bombe getroffen hatte ${ }^{35}$.

Die Katastrophe von Diên Biên Phu im Mai ließ den Ruf nach einer nationalen Atomstreitmacht in Paris lauter denn je erschallen. Im Gegensatz zur Regierung vertrat der Generalstab indes die Auffassung, die notwendigen Beschlüsse nicht mit der Europäischen Verteidigungsgemeinschaft in Einklang bringen zu können, zumal Artikel 107 des Vertrages ja ein Atomwaffenverbot für sogenannte exponierte Gebiete vorsah. Als Ersatzlösung schlug er Ende Juni eine multilaterale Verteidigungsorganisation auf der Basis des Brüsseler Paktes vor ${ }^{36}$. Im Quai d'Orsay fing man an, darüber nachzudenken, wie die Bundesrepublik in eine Kooperation eingebunden werden könnte, bevor die Fesseln des Pariser Vertrags fielen. Sauvagnargues schlug dazu im Juli vor, auf der Regierungsebene die gemeinsame Produktion von Flugzeugen in Nordafrika in einem europäischen Rahmen anzugehen, wobei er sich in einem späteren Stadium auch die Zusammenarbeit bei Raketen und Nuklearwaffen vorzustellen imstande war $^{37}$.

Regierungschef Mendès France war nicht nur über die Nuklearpläne der Generale eingeweiht, er wußte auch um deren Einschätzung von der Inkompatibilität zwischen der EVG und der Atombombe und kannte möglicherweise auch die Alternativvorstellungen des Generalstabs. Sowohl Colonel Ailleret, Chef du com-

\footnotetext{
Vgl. Bariéty, Frankreich und das Scheitern der EVG, S. 109; Coutrot, Politique atomique, S. 310. Vgl. Maier, Auseinandersetzungen, S. 135-139; Peter, Abschrecken; Rosenberg, Origins.

34 Zur NATO-Ratstagung vom 14.-16. 12. 1953 s. die Dokumente in: FRUS 1952-1954, Bd. 5, S. 454-481; Schlußkommuniqué, 16. 12. 1953, in: EA 1954, S. 6309 f.; Maier, Auseinandersetzungen, S. $170 \mathrm{f}$.

35 S. Ailleret, Aventure atomique, S. 164-175; Bougeard, René Pleven, S. 260; Doise/Vaïsse, Diplomatie, S. 597; Scheinman, Atomic energy policy, S. 106-109.

36 Vgl. Kap. III.4.3.

37 S. Aufzeichnung Sauvagnargues, 10. 7. 1954, in: BDFD II, S. 219-221.
} 
mandement des armées spéciales, chargé de la recherche nucléaire militaire, als auch Colonel Gallois, die massiv für den Bau der Bombe eintraten, hatten in diesen Wochen zahlreiche Unterredungen mit ihm. Eine wichtige Mittlerrolle zwischen dem Président du Conseil, dem CEA und den Militärs spielte der Leiter des Kabinetts von Mendès France im Matignon, André Pelabon. Am 3. 8. erhielt er vom Staatssekretär für wissenschaftliche Forschung und technischen Fortschritt, Longchambon, ein Programm über die zivile und militärische Anwendung der Atomkraft. Zehn Tage später sandte der Leiter der französischen Delegation in der Ständigen NATO-Ratsgruppe, General Valluy, dem Chef d'Etat-Major Général des Forces Armées, General Guillaume, einen streng geheimen Bericht über die jüngsten Entscheidungen der NATO im Rüstungsbereich, der als Kopie am 20. 8. auch an Pelabon und direkt an Mendès France ging. Valluy sah die NATO vor grundlegenden Änderungen der Verteidigungskonzeption, die der Atomwaffe die entscheidende Rolle in zukünftigen Militärkonflikten übertrügen. Um sich sicherheitspolitisch nicht völlig von den USA abhängig zu machen, riet er Europa zum Aufbau eines eigenen Atomarsenals ${ }^{38}$. Am 14. 8. wandte sich der Ständige Vertreter Frankreichs im NATO-Rat, Alphand, an Mendès France und setzte ihn über einen der Nordatlantischen Allianz zur Prüfung vorliegenden Bericht über die Revision der strategischen Konzeption aufgrund der Einbeziehung neuer Waffen in Kenntnis. Kernelement des Rapports war „une riposte atomique occidentale automatique" gegen jede Aggression, die die Sowjetunion der Gefahr eines nuklearen Gegenschlags aussetze und damit einer „modification révolutionnaire" der europäischen Sicherheit den Weg ebne ${ }^{39}$. Sechs Tage später führte Valluy Pelabon die Tragweite der strategischen Änderungen persönlich vor Augen: „S'il y a attaque russe atomique ou non le Général Gruenther entend utiliser les représailles atomique immédiates. " 40

In einem Notenwechsel mit dem Quai d'Orsay vertrat der französische Generalstab des Heeres am 11. 9., notabene nach dem Scheitern der EVG, die Auffassung, die von ihm Ende Juni ins Auge gefaßte europäische Verteidigungsorganisation solle Atomwaffen besitzen. Im Klartext bedeutete dies den Anspruch Frankreichs auf einen Platz im Kreis der nuklearen „Haves“, den man der Bundesrepublik aber dezidiert verwehrte. Da man die Doppelbelastung einer atomaren Aufrüstung und einer der Bundesrepublik ebenbürtigen konventionellen Bewaffnung nicht allein tragen konnte, empfahlen die Militärs die Schaffung einer integrierten europäischen Atomstreitmacht im Rahmen eines atlantischen Atompools. Die Pläne de Gaulles aus dem Jahre 1958 vorwegnehmend, dachten sie an ein strategisches Dreierdirektorium, dessen Berechtigung ungeachtet der fehlenden Nuklearkapazität aus der Weltmachtstellung Frankreichs abgeleitet wurde ${ }^{41}$.

$38 \mathrm{Vgl}$. Kap. III.4.3.

39 IPMF, DPMF, Dossier CED, Carton 2, Alphand an Mendès France, Aufzeichnung, 14. 8. 1954, Personnel et très secret.

40 Ebd., Unterredung zwischen Valluy und Pelabon vom 20. 8. 1954, Très secret; s.a. Kap. III.4.3.

41 S. Kap. III.4.4. 


\section{Das Rüstungspoolprojekt}

Mendès France nahm sich dieses Vorhabens an und entwickelte den Plan eines europäischen Rüstungspools, dem nicht nur die Errichtung neuer Fabriken unterstellt werden, sondern auch die Entgegennahme und Verteilung der US-Militärhilfe für Westeuropa obliegen sollte. Aufgrund der internationalen Kritik versuchte der Regierungschef Bundeskanzler Adenauer Ende September mit dem Argument zu gewinnen, eine solche wirtschaftliche Verklammerung entfalte eine große Schubwirkung für eine Wiederbelebung des Europagedankens. Bonn stand dem Pariser Werben aber sehr skeptisch gegenüber, da das System nicht völlig gleichberechtigt aufgebaut wirkte und die Frage der britischen Beteiligung sowie die Verteilung der amerikanischen Militärhilfe ungeklärt waren ${ }^{42}$. Um die Deutschen geneigter zu stimmen, signalisierten die Franzosen die Bereitschaft, den Rüstungspool in ein umfassendes Kooperationskonzept einzubetten. Abermals stand die Luftfahrtindustrie im Vordergrund, wobei auch der Atomsektor nie völlig aus dem Blickwinkel der Verantwortlichen in Paris verschwand. Man war sich nach der Londoner Viermächtekonferenz nämlich nur zu bewußt, daß die selbst auferlegten Fesseln der Bonner Republik nicht überbewertet werden durften. Adenauer hatte gewisse Beschränkungen akzeptiert, doch die Erfahrung lehrte, „à ne pas leur attacher une valeur autre que de retardement ${ }^{\text {“4 } 43}$.

Genährt von sicherheitspolitischen und wirtschaftlichen Ambitionen, ging es Paris zunächst um eine breite technische Zusammenarbeit in Heer und Luftwaffe ${ }^{44}$. „Le réarmement de l'Allemagne“, so formulierte Mendès France ein Hauptkalkül dieser Kooperationspolitik, „paraîtra aux Français comme beaucoup moins dangereux si les Allemands s'approvisionnent en France d'une partie de leurs armes et s'ils se dirigent [...] vers la création d'usines de fabrication francoallemandes, qui pourraient être situées par exemple dans certains de nos territoires d'outre-mer." 45 Dabei machten die Franzosen intern keinen Hehl daraus, daß diese Absichten auch eine antienglische Spitze besaßen. Mit dem Angebot zur deutsch-französischen Kooperation wollten sie dem britischen Streben begegnen, der eigenen Luftfahrtindustrie die Vorherrschaft auf dem deutschen Markt zu sichern $^{46}$. Dabei dachte man sogar daran, der Bundesrepublik Waffensysteme anzubieten, „dont les caractéristiques sont encore tenues secrètes à l'égard des Anglais et des Américains“. Dazu gehörten neben den Übungsflugzeugen „Morane“ und "Fouga" auch geheime Prototypen wie "Gerfault", „Trident" und „Leduc"47, nicht aber an die von Bonn gewünschte Überlassung einer Lizenz zum Bau der

42 S. ebd.

43 Aufzeichnung des MAE, o.D., in: DDF 1955, Bd. 1, S. 546-551, hier S. 549.

44 S. AMAE, Europe 1944-1960, Allemagne, Bd. 208, Bl. 1-4, Aufzeichnung der Abt. für wirtschaftliche und finanzielle Angelegenheiten im MAE, 13. 10. 1954, Très secret (Konzept GC); s.a. Bossuat, Armements, S. 604.

45 Mendès France an François-Poncet, Tel. 4569/93, 28. 12. 1954, Réservé, in: DDF 1954, S. 975 f., hier S. 976.

46 S. Aufzeichnung Clauzel, 11. 10. 1954, Très secret, in: BDFD II, S. 356.

47 AMAE, Europe 1944-1960, Allemagne, Bd. 208, Bl. 1-4, Aufzeichnung der Abt. für wirtschaftliche und finanzielle Angelegenheiten im MAE, 13. 10. 1954, Très secret (Konzept GC). Zum französischen Luftwaffenprogramm vgl. BA, NL Blankenhorn, Bd. 80, Bl. 166-171, Seeger-Kelble an Blankenhorn, Aufzeichnung 70-00-06800/57, 28. 10. 1957; s.a. allgemein Martel/Frémeaux/Bénichou/Carlier, La France, S. 56-58. 
„Mystère $B$ “ von Dassault. Paris zielte nicht nur auf Lieferungen eigener Maschinen und Geräte, sondern auch auf gemeinsame Forschungsprojekte und die Konstruktion von Rüstungswerken für die Luftwaffe. Als die Bundesrepublik mit der Londoner Schlußakte vom 3. 10. das Recht zur Errichtung eigener Flugzeugfabriken erhielt, plädierte der Quai d'Orsay dagegen, derartige Unternehmen in Nordafrika zu bauen, offenbar weil er keine Chance sah, dafür die Zustimmung der Deutschen zu gewinnen. Demgegenüber blieb man von seiten des Luftfahrtministeriums bei diesem Standort aus Sorge um eine etwaige Bombardierung solcher Fabriken in Deutschland ${ }^{48}$.

Von François-Poncet über die französischen Absichten informiert, reagierte Adenauer sehr aufgeschlossen. Die Kooperation in der Luftfahrtindustrie schien ihm vom psychologischen wie politischen Aspekt fruchtbar zu sein. Daher begrüßte er auch die französische Einladung an deutsche Spezialisten zu Materialvorführungen ${ }^{49}$. Weit weniger interessiert zeigte sich die Bundesregierung hingegen an dem von Frankreich Mitte Oktober erneut in die Diskussion gebrachten Rüstungspool, der für die Produktion und Standardisierung der Rüstungsgüter, für die Aufstellung von Rüstungsprogrammen, die gemeinsame Beschaffung der Waffen und Verteilung der Militärhilfen, Kontrolle der Rüstungsindustrie und Bau gemeinsamer Rüstungswerke zuständig sein sollte. Da nach den Erfahrungen auf der Londoner Außenministertagung von den Briten und den BENELUXStaaten wenig Sympathie zu erwarten war, unternahmen die Franzosen im Vorfeld der Pariser Konferenz einen Anlauf, die Unterstützung der Deutschen mit dem Hinweis auf den europapolitischen Impetus dieses Projektes zu ködern ${ }^{50}$. Der Erfolg war äußerst mäßig. Im Bundeswirtschaftsministerium wurde die Idee des Pools höchst kritisch betrachtet. Erhard begrüßte zwar Vereinbarungen über Auswahl und Standardisierung der Rüstung, wollte diese Aufgabe aber bei der NATO aufgehoben wissen. Für eine gemeinsame Beschaffung sah er nach dem Scheitern der EVG „kein[en] Raum“ mehr. Die von Frankreich angestrebte Kontrolle der Rüstungsindustrie und die Genehmigungspflicht war für ihn „völlig unannehmbar". Ohnehin wurde er den Verdacht nicht los, daß Paris die Schaffung eines zusätzlichen Kontrollinstruments bloß in europäische Gewänder hüllte. Eine supranationale Rüstungsgemeinschaft unter französischer Führung bot ein ideales Instrument zur Schwächung des deutschen Industriepotentials. Allenfalls denkbar war für ihn die Schaffung gemeinsamer Rüstungswerke, sofern der Grundsatz der Gegenseitigkeit gewahrt bliebe ${ }^{51}$. Das Auswärtige Amt mochte sich dem französischen Plan hingegen nicht entziehen, sofern die Organisation möglichst locker, die Hinzuziehung der BENELUX-Staaten und Italiens gesichert und die Assoziation Großbritanniens gewährleistet sein würde 52 .

Entsprechend schwierig gestaltete sich die Behandlung des Themas während der sechsstündigen Unterredung zwischen Mendès France und Adenauer am

48 S. Aufzeichnung Clauzel, 11. 10. 1954, Très secret, in: BDFD II, S. 356-358.

49 S. AMAE, Europe 1944-1960, Allemagne, Bd. 502, B1. 36, MAE an François-Poncet, Tel. 3282/83, 7. 10. 1954; ebd., 43f., François-Poncet an MAE, Tel. 4923/25, 8. 10. 1954; ebd., Bd. 208, Bl. 5, ders. an dass., Tel. 5114/16, 15. 10. 1954, Réservé, Secret; Bossuat, Armements, S. 604.

so S. PA, Abt. 3, AZ 230-07, Bd. 8, Hausenstein an AA, Tel. 622, 15. 10. 1954; Thoß, Beitritt, S. 53.

51 Erhard an Adenauer, 18. 10. 1954, in: BDFD II, S. 362-364, hier S. 362 u. 363.

52 S. Aufzeichnung des AA, 18. 10. 1954, Geheim, in: ebd., S. 367; Kap. III.4.4. 
19. 10. auf Schloß La Celle-St.-Cloud. Aus der Sicht des Président du Conseil sollte der Waffenpool neben einer rigiden Kontrollfunktion die Verteilung der von den USA gelieferten Waffen und die Überwachung der Rüstungsproduktion auf dem Kontinent übernehmen. Außerdem verlangte er eine Zentralbehörde für die Aufstellung der Rüstungsprogramme und der Standardisierung. Der Kanzler billigte letzteres, die Schaffung einer Stelle zur Abstimmung des Bedarfs und der Produktion sowie gemeinschaftliche Rüstungsunternehmen, von der zentralen Agentur wollte er aber nichts wissen, stimmte aber zu, den Gesamtkomplex innerhalb der WEU weiterzubehandeln ${ }^{53}$. Zwei Tage später drängte Mendès France gegenüber den Außenministern der neun Mächte auf die Aufnahme entsprechender Beratungen schon zum 1. 12. Doch die Furcht vor einer junktimsartigen Verknüpfung zwischen den Pariser Verträgen und dem Rüstungspool rief den Widerstand der Partner hervor. Der französische Regierungschef willigte daher in eine Resolution ein, derzufolge die Verhandlungen erst am 17.1.1955 beginnen und zunächst auf Arbeitsebene geführt werden sollten ${ }^{54}$.

Je stärker sich die Aussicht auf das Überwachungsinstrument des Rüstungspools im Ungefähren verflüchtigte, desto stärker entwickelte sich das französische Interesse an einer direkten deutsch-französischen Zusammenarbeit. Ende Oktober fand es in einer Vorführung französischer Flugzeuge auf dem französischen Übungsgelände bei Lahr, einen Monat später in der Präsentation von Panzern bei Mailly ersten Niederschlag55. Presseberichte, wonach Teile der deutschen Industrie große Erwartungen in eine gemeinsame Waffenproduktion auf französischem Gebiet setzten, andere Unternehmen hingegen an eine rein deutsche Produktion dachten und dazu auf die Hilfe der Angelsachsen hofften ${ }^{56}$, taten ein übriges. Beide Faktoren reichen aber nicht aus, um zu erklären, wieso Frankreich sich nun mehr und mehr auf eine tiefgreifende Kooperation mit der Bundesrepublik im Verteidigungsbereich einließ. Wesentlichen Einfluß auf die französische Entscheidungsfindung hatte wohl der unmittelbar nach den Pariser Konferenzen eingeleitete Einstieg in die militärische Nuklearforschung, die am 26. 12. in den Beschluß mündete, „à lancer un programme secret d'études et de préparation d'un prototype d'arme nucléaire et d'un sous-marin atomique" 57 . Frankreich reagierte damit auch auf die Mitte Dezember auf der NATO-Konferenz abgesegnete Nuklearisierung der Bündnisstrategie, die den europäischen Verbündeten im Sinne der Schwert-Schild-Doktrin die Aufgabe zuwies, ausreichende konventionelle Streitkräfte bereitzustellen, während

53 S. BA, NL Blankenhorn, Bd. 35, Bl. 313-325, Unterredung zwischen Adenauer und Mendès France vom 19. 10. 1954, im Auszug in: BDFD II, S. 368-372; PA, Abt. 3, AZ 210-01/22, Bd. 4, Blankenhorn u. Hausenstein an von Welck, Tel. 634, 20. 10. 1954, Vertraulich, Citissime; Soutou, L'alliance incertaine, S. 26-30.

54 S. Sitzung der Neun-Mächte-Konferenz vom 21. 10. 1954, in: DDF 1954, Annexes, S. 407-448; US-Delegation an Dep. of State, Tel. Secto 8, 22.10. 1954, Secret, in: FRUS 1952-1954, Bd. 5, S. 1415-1417; dies. an dass., Tel. Secto 9, 22. 10. 1954, Secret, in: ebd., S. 1417-1419; Kap. III.4.4.

55 S. AMAE, Europe 1944-1960, Allemagne, Bd. 502, Bl. 84 f., François-Poncet an MAE, Tel. 5355, 30. 11. 1954; Bossuat, Armements, S. 604.

56 S. AMAE, Europe 1944-1960, Allemagne, Bd. 208, Bl. 7 f., Bérard an Mendès France, Nr. 2758/ EU, 30. 11. 1954, Secret; Französische Dienststelle im Militärischen Sicherheitsamt, Aufzeichnung Nr. 4088/HC/OMS/COM/S, 27. 11. 1954, Secret, in: BDFD II, S. 391-395.

57 Goldschmidt, Rivalités, S. 207; s.a. ders. Complexe atomique, S. 146 f.; Ailleret, Aventure atomique, S. 142f. u. 151-178; Scheinman, Atomic energy policy, S. 111-116; Soutou, Politique nucléaire, S. 323-330. 
die USA für den nuklearen Schutz sorgen sollten. Da aber noch keine konkreten Umsetzungsbeschlüsse der Direktive MC 48 existierten, gab die Bundesregierung der faktischen Integration in die NATO durch die unverzügliche Aufstellung der zwölf Divisionen unbedingten Vorrang vor einer Diskussion über etwaige Anpassungsmaßnahmen. Um dieses Ziel zu erreichen, mußte sie natürlich einerseits ihre Verpflichtungen einhalten. Andererseits war es aber auch notwendig, daß die Widersprüchlichkeit zwischen der Nuklearisierungsentscheidung der NATO und den weiterhin gültigen konventionellen Planungen für den auf drei Jahre angelegten Zeitraum der Aufstellung der Bundeswehr gültig blieb ${ }^{58}$. „Europa lebe sicher unter dem Schild der amerikanischen Atomwaffen, und diese Sicherheit könne noch erhöht werden, wenn ein Teil dieses Schilds selbst in Europa sei "59, hatte Adenauer im Juni 1953 dem amerikanischen General Ridgway gesagt. Daran hatte sich im Laufe des letzten Jahres für die Bundesrepublik wohl nichts geändert.

Frankreich bemühte sich nun darum, die sicherheitspolitische Lage des östlichen Nachbarn in vielfältiger Hinsicht zum eigenen Vorteil zu nutzen: wirtschaftlich durch den Absatz eigener Rüstungsgüter für den Aufbau der Bundeswehr, militärisch durch die Nutzbarmachung des technischen deutschen Know how auch im Atombereich. Anfang November beauftragte Mendès France Longchambon, konkrete Vorschläge zur deutsch-französischen Nuklearzusammenarbeit auszuarbeiten, um die wissenschaftlichen Kenntnisse des Nachbarn in die eigenen Forschungen einfließen zu lassen und Einblicke über ihre technologischen Fortschritte zu bekommen 60 .

Gleichzeitig forcierte Paris die Bemühungen um den Rüstungspool. Dazu arbeitete der Quai d'Orsay auf Weisung des Außenministers den Entwurf eines Memorandums über die Standardisierung und die Rüstungsproduktion aus, das zu der von Frankreich bereits auf der Londoner Konferenz vertretenen, aber von den übrigen Teilnehmern abgelehnten Linie zurückkehrte ${ }^{61}$. Am 3. 1.1955 unterbreitete die Regierung den WEU-Partnern die offizielle Fassung des langen, aber dennoch bisweilen recht vagen Dokuments, über das man ausschließlich im Rahmen der WEU unabhängig von der NATO zu beraten gedachte. Frankreich regte darin die Schaffung einer Behörde an, die für die Gewährleistung der Standardisierung, die jährliche Aufstellung von Rüstungsprogrammen und deren Ausführung durch Vergabe der Produktionsaufträge sowie die Planung und Koordinierung der Investitionen verantwortlich sein sollte. Angesichts der nicht sehr weit vorangeschrittenen Standardisierung sah man eine bis Ende 1956 dauernde Vorlaufzeit sowie ein dann einsetzendes definitives Regime vor ${ }^{62}$.

In Bonn stieß das Pariser Projekt noch immer auf unterschiedliche Reaktionen, wobei das Bundeswirtschaftsministerium ihm weiterhin skeptischer gegenüber-

58 Vgl. Fischer, Abschreckung, S. 278-280; ders. Atomenergie, S. 158-163.

59 PA, Abt. 2, Bd. 973, Unterredung zwischen Adenauer und Ridgway vom 15.6. 1953, 16. 6. 1953, Geheim; s.a. Dulles an Büro der Hohen Kommission Bonn, Tel. 230, 17. 7. 1953, Top secret, Priority, in: FRUS 1952-1954, Bd. 7, S. 486 f.; Maier, Internationale Auseinandersetzungen, S. $137 \mathrm{f}$.

60 S. Mendès France an Longchambon, Nr. 137/PAN, 5. 11. 1954, Secret, in: BDFD II, S. $379 \mathrm{f}$.

61 Vgl. Guillen, Frankreich und die NATO-Integration, S. 442; Kap. III.4.3.

62 S. Memorandum der französischen Regierung, 3. 1. 1955, in: DDF 1955, Annexes, Bd. 1, S. 245-251; PA, B 14, Ref. 211, Bd. 58, Bl. 74, von Welck an Blankenhorn, Vermerk 3.223-00.31575/54, 13.12. 1954, Sofort; AdG 1955, S. 4951 A; Calandri, Western European Union Armament Pool, S. 53 f. 
stand als das Auswärtige Amt. Die Beamten in der Koblenzer Straße hofften auf einen vom Pool ausgehenden neuen Schub für die europäische Integration und wünschten der Rüstungsagentur über die Standardisierung hinaus weitere Kompetenzen zu verleihen. Einvernehmen herrschte zwischen den Ministerien aber darüber, das Rüstungsamt strikt von der Rüstungskontrollbehörde der WEU zu trennen, da sie dem Pool weder die Kontrolle noch die Beschaffung von Rüstungsgütern zu übertragen gedachten ${ }^{63}$. Das Bundeswirtschaftsministerium empfahl daher, die französische Forderung nach Bildung einer Rüstungsagentur nicht en bloc zurückzuweisen, sondern durch Gegenvorschläge zu ersetzen, zumal die französischen Überlegungen zur Rüstungsgemeinschaft hinsichtlich der gemeinsamen Auswahl und Standardisierung der Ausrüstung der Streitkräfte und der Koordinierung der nationalen Rüstungsprogramme akzeptiert werden konnten. Außerdem mußte die Bundesregierung natürlich sorgsam darauf achten, daß der Rüstungspool nicht zum politischen Handelsobjekt für die Ratifizierung der Pariser Verträge würde ${ }^{64}$.

Dies wurde im Auswärtigen Amt gewiß genauso gesehen. Ansonsten stimmten die Beamten von der Koblenzer Straße aber nur zum Teil mit ihren Kollegen aus Duisdorf überein. Das Auswärtige Amt begrüßte den Plan, soweit es sich um eine integrierte Organisation handelte, kritisierte ihn, sofern Frankreich ein neues Kontrollorgan bilden und seiner Industrie Vorteile verschaffen wollte. In Übereinstimmung mit dem Wirtschaftsministerium pochte man darauf, die Standardisierung nur nach Abstimmung mit der NATO durchzuführen. Uneinigkeit herrschte zwischen den beiden Ministerien dagegen in bezug auf die Beschaffung, da von seiten der Wirtschaftspolitiker vor den Gefahren dirigistischer Tendenzen durch die supranationale Behörde gewarnt wurde, während die Diplomaten diesbezüglich weniger Argwohn hegten ${ }^{65}$.

Im Gegensatz zu den BENELUX-Staaten, die das französische Memorandum vom 3. 1. wegen der dirigistischen Regeln des sogenannten Régime définitif sofort verwarfen ${ }^{66}$, riet das Auswärtige Amt in einer ersten Stellungnahme zu differenziertem Urteil. Könne der Rüstungspool in ein europäisches Gesamtsystem eingefügt werden, fragten sich die Beamten? Führe das Kontrollsystem über den positiven Effekt der "gegenseitigen Abhängigkeit der Teilnehmerstaaten“ zu einer Lähmung des eigenen Wehrbeitrages? Greife Frankreich gerade deshalb auf supranationale Elemente zurück, um einen Grundsatzstreit zu entfachen, der den weiteren Ratifikationsprozeß störe?67 Ein Kompromiß zwischen den französischen Wünschen und der schroffen Haltung der BENELUX-Staaten schien nur dann möglich, wenn Paris auf seine „Sonderziele“ verzichtete und davon Abstand nahm, den Pool „in den Dienst einer indirekten Rüstungskontrolle mit Beschrän-

63 S. PA, B 14, Ref. 211, Bd. 58, Bl. 43-45, Heiser an Ophüls, Aufzeichnung, 18. 11. 1954.

64 S. Krautwig an Westrick, Aufzeichnung IV A 6-36391/54, 30.11. 1954, Vertraulich, in: BDFD II, S. 398-400.

65 S. PA, B 14, Ref. 211, Bd. 58, Bl. 57-63, Ressortbesprechung vom 3. 12. 1954, 215.226-04/2150/54, 7. 12. 1954; Thoß, Beitritt, S. 97.

66 S. PA, B 14, Ref. 211, Bd. 41, Bl. 22-24, von Etzdorf an AA, 223-32-1026/55, 7. 1. 1955, Geheim; Calandri, Western European Union Armament Pool, S. 55f.; Thoß, Beitritt, S. 98.

67 PA, B 14, Ref. 211, Bd. 41, Bl. 25-34, „Einige Bemerkungen zum französischen Vorschlag eines ,Rüstungspools“", Januar 1955. 
kung der Rüstungskapazitäten" $z u$ stellen, was ihm schon auf der Londoner Konferenz nicht gelungen war ${ }^{68}$.

Erhard behagte der den politischen Aspekt des französischen Konzeptes herausstellende Ansatz des Auswärtigen Amtes ganz und gar nicht. In einem Schreiben an Adenauer begrüßte er zwar den Wunsch nach bilateraler Rüstungszusammenarbeit und sah in den Vorstellungen von Mendès France auch „echte Ansatzpunkte". Er drängte aber darauf, bei der am 17. 1.1955 beginnenden Expertenkonferenz über "Rüstungsproduktion und -standardisierung" die wirtschaftspolitischen und militärtaktischen Aspekte den „absoluten Vorrang“ einzuräumen ${ }^{69}$. Ohne von dieser Argumentation vollends überzeugt zu sein, beauftragte der Kanzler seinen Wirtschaftsminister nolens volens mit der Delegationsführung für die Sachverständigenkonferenz ${ }^{70}$. Am 11. 1. legte Erhard ihm daraufhin den Entwurf eines eigenen Memorandums vor, der in Übereinstimmung mit den von ihm konsultierten Gewerkschaften und Industrieverbänden ${ }^{71}$ das französische Dokument in "vielen Punkten“ als unannehmbar bezeichnete ${ }^{72}$. Obwohl Mendès France Adenauer drei Tage danach in Baden-Baden eindringlich auf die Bedeutung des Projektes für Frankreich hinwies und sich zu Abänderungen seines Konzepts durchaus bereit zeigte, setzte sich Erhard durch. Die Hoffnung des Président du Conseil auf eine gemeinsame oder doch zumindest „analoge Stellungnahme“ blieb unerfüllt ${ }^{73}$. Denn das die deutschen Vorstellungen zusammenfassende, inzwischen mit Hallstein abgestimmte Memorandum des Bundeswirtschaftsministers arbeitete trotz des Bemühens um Gemeinsamkeit die unterschiedlichen Auffassungen klar heraus. Zwar sprach sich Erhard für eine weitreichende Kooperation der beteiligten Staaten, die Standardisierung der Waffensysteme, die Harmonisierung der Rüstungsprogramme und den Bau gemeinsamer Rüstungsfabriken aus. Doch standen seine gesamten Ausführungen unter dem lauten Credo, die Rüstungsindustrie nach den Prinzipien der freien Marktwirtschaft funktionieren und die Zusammenarbeit im Wege der Konsultation und des Meinungsaustauschs erfolgen zu lassen. Ein übernationales Koordinierungsgremium hielt er für unzweckmäßig, die zentrale Vergabe von Rüstungsaufträgen nicht mit dem WEU-Vertrag vereinbar ${ }^{74}$. Immerhin verständigten sich beide Regierungen auf die Beibehaltung des Grundgedankens einer Koordination der westeuropäischen Rüstungsmaßnahmen, die Hallstein am 19. 1. vor dem Bundeskabinett als eine „Art gemeinsamen Markt für die Rüstungsproduktion" bezeichnen sollte75.

68 BA, NL Blankenhorn, Bd. 38a, Bl. 132-137, Aufzeichnung der Abt. $2 / 21$ im AA, 226-04/1/55, 6. 1. 1955, Geheim.

69 PA, B 14, Ref. 211, Bd. 41, Bl. 132-134, Erhard an Adenauer, IV A6-36623/54, 10. 12.1954.

70 Ursprünglich hatte der Kanzler allem Anschein nach Ophüls mit dieser Aufgabe betrauen wollen (Noack, Scheitern, S. 157f.).

71 Vgl. Thoß, Beitritt, S. $99 \mathrm{f}$.

72 BA, NL Blankenhorn, Bd. 38a, Bl. 81 f., Erhard an Adenauer, 11. 1. 1955; Thoß, Beitritt, S. 99.

73 BA, NL Blankenhorn, Bd. 39, Bl. 9-22, Unterredung zwischen Adenauer und Mendès France vom 14. 1. 1955, Geheim, im Auszug in: BDFD I, S. 198; s.a. Unterredung zwischen dens. vom 14. 1. 1955, in: DDF 1955, Annexes, Bd. 1, S. 229-235; Calandri, Western European Union Armament Pool, S. 55.

74 S. Memorandum Erhards, 14. 1. 1955 (Übersetzung), in: DDF 1955, Annexes, Bd. 1, S. 252-255; AMAE, Europe 1944-1960, Allemagne, Bd. 388, Bl. 53, de Margerie an Rom, Tel. 255/59, 15.1. 1955, Priorité.

75 Zitiert nach: Thoß, Beitritt, S. 100. 
Zwei Tage zuvor war die Konferenz der WEU-Arbeitsgruppe über „Rüstungsproduktion und -standardisierung “ eröffnet worden. Schon die ersten Sitzungen ließen erkennen, daß Frankreich weitgehend isoliert war ${ }^{76}$. Erhard nutzte diese Schwäche sofort aus und erklärte den Dirigismus der französischen Pläne für unvereinbar mit einer liberalen Wirtschaftsordnung. Trotz mehrtägiger Unterbrechung traten die Beratungen nach der Wiederaufnahme Ende Januar auf der Stelle. Am 2. 2. vertagte sich die Konferenz erneut und setzte lediglich einen Ausschuß zur Prüfung des gegenwärtigen Zustandes der Standardisierung und Bedarfsdekkung von Rüstungsgütern ein ${ }^{77}$.

Der französische Versuch, Erhard für dieses wenig erfreuliche Ergebnis zur Verantwortung zu ziehen, wurde von Adenauer unter Hinweis auf die starre Haltung der Briten und Holländer sofort zurückgewiesen ${ }^{78}$. Die Franzosen hielten sich daher mit weiterreichender Kritik zurück, zumal es in parallel geführten Gesprächen mit deutschen Experten bemerkenswerterweise zu einer „erhebliche[n] Annäherung “ der Standpunkte kam. Paris erkannte die Bonner Hauptdesiderata an: Rüstungspool und Ratifizierungsdebatte im Senat sollten nicht durch ein Junktim verbunden, die amerikanische Verteidigungshilfe ausgeklammert und der deutschen Industrie keine über das Pariser Vertragswerk hinausgehenden Kontrollen auferlegt werden. Dafür erklärte sich Bonn bereit, auf einen ständigen westeuropäischen Rüstungsausschuß für Standardisierung und Produktion als Endziel der Verhandlungen hinzuwirken, der freilich lediglich empfehlende und keine bindenden Entscheidungen treffen durfte ${ }^{79}$.

Der Sturz von Mendès France Anfang Februar legte die Rüstungspool-Angelegenheit wochenlang auf Eis. Anfang April stimmte die neue Regierung unter Edgar Faure einem britischen Kompromißvorschlag zu, der einen ständigen WEU-Rüstungsausschuß vorsah, dessen Vorschläge sich aber in engster Absprache mit der NATO zu bewegen hatte und der ohne jegliche supranationale Kompetenzen bleiben sollte ${ }^{80}$. Am 7. 5. beschloß der Rat der WEU auf seiner ersten Sitzung, diesen Empfehlungen zu folgen ${ }^{81}$.

76 S. Bérard, Ambassadeur, Bd. 2, S. 613 f., Tb. 18. 1. 1955; Calandri, Western European Union Armament Pool, S. 58.

7 S. Thoß, Beitritt, S. 101; vgl. PA, B 14, Ref. 211, Bd. 41, Bl. 153-155, Heiser an von Maltzan, Aufzeichnung, 24.1. 1955; ebd., Bl. 156-163, von Maltzan an AA, 2. 2. 1955; Garnier an Faure, Nr. 159, 2. 2. 1955, Confidentiel, in: DDF 1955, Bd. 1, S. 145-147; AdG 1955, S. 4990K; Calandri, Western European Union Armament Pool, S. 58; Hentschel, Ludwig Erhard, S. 222 f.; Noack, Scheitern, S. 160.

78 S. AMAE, Europe 1944-1960, Allemagne, Bd. 388, Bl. 144-146, François-Poncet an MAE, Tel. 461/69, 4. 2. 1955, Réservé; ders. an Faure, Tel. 470, 4. 2. 1955, in: DDF 1955, Bd. 1, S. 150 f.; Aufzeichnung von Merkatz, 3. 2. 1955, in: BDFD III, S. 409-411.

79 PA, B 14, Ref. 211, Bd. 41, Bl. 171-173, von Maltzan an AA, 4. 2. 1955; Coignard an Seydoux, Nr. 505/HC/OMS/COM, 9. 3. 1955, Confidentiel, nebst Aufzeichnung vom 23. 2. 1955, Confidentiel, in: DDF 1955, Bd. 1, S. 303-308; Calandri, Western European Union Armament Pool, S. 59-61; Thoß, Beitritt, S. 101 u. 103.

80 S. Coignard an Seydoux, Nr. 505/HC/OMS/COM, 9. 3. 1955, Confidentiel, nebst Aufzeichnung vom 23. 2. 1955, Confidentiel, in: DDF 1955, Bd. 1, S. 303-308; PA, Abg.-Liste Ref. 204, Bd. 12, von Maltzan an Hallstein, Aufzeichnung 215.226-04/612/55, 7.4. 1955; Guillen, Frankreich und die NATO-Integration, S. 443; Thoß, Beitritt, S. 103.

81 S. PA, B 14, Ref. 211, Bd. 41, Bl. 226-232, Ophüls an Diplomatische Vertretungen, 86-05-2, 27. 5. 1955, Reinkonzept, Cessat; Noack, Scheitern, S. 162. 
"Cauchemar d'un programme atomique germano-américain dépassant le programme français"

Frankreich pflichtete der Entscheidung bei, obwohl sie natürlich weit von dem entfernt war, was man ursprünglich intendiert hatte. Man konnte es um so leichter tun, als sich die Rahmenbedingungen dieses Projektes mit dem Regierungswechsel in Frankreich von Mendès France zu Faure und den in der Sechsergemeinschaft diskutierten Plänen über eine Europäische Atomgemeinschaft erheblich geändert hatten. Gewiß, mit Verteidigungsminister Pierre Koenig und Gaston Palewski, dem beigeordneten Minister im Matignon für Atom- und Rüstungsfragen, gab es in der neuen Regierung starke Befürworter einer französischen Atomwaffe. Der Regierungschef selbst aber zögerte noch. Zwar gab er Mitte April auf einer Pressekonferenz die Entscheidung Frankreichs zum Aufbau einer Atommacht bekannt, beschränkte sie aber ausdrücklich auf den zivilen Sektor ${ }^{82}$. Für den militärischen Bereich schloß er sich der Meinung jener an, die die Entscheidung für zwei bis drei Jahre verschieben wollten und zunächst einmal an den Aufbau einer nuklearen Infrastruktur im Land dachten ${ }^{83}$. Dies hielt Koenig und Palewski aber nicht davon an, Mitte Mai mit Wirtschafts- und Finanzminister Pflimlim zu vereinbaren, Haushaltsmittel aus dem Wehretat dem Commissariat à l'Energie Atomique zur Verfügung zu stellen ${ }^{84}$.

Die militärische Führung dachte derweil insgeheim über die Einbeziehung der Bundesrepublik in ein militärisches Atomprogramm nach. Ailleret etwa vertrat die Auffassung, daß Frankreich mit seinen beschränkten industriellen Ressourcen vor allem im chemischen Bereich nur dann eine größere Nuklearindustrie aufbauen könne, wenn es deutsche Mittel anzapfte. Ihm schwebte eine echte europäische Atomrüstung vor, die neben dem integrationspolitischen Schub aus seiner Sicht den Vorteil besaß, die Existenz einer europäischen Armee zu sichern, die durch sich selbst und nicht bloß als Infanterie einer Atommacht Bedeutung gewänne ${ }^{85}$. Anläßlich zweier interministerieller Sitzungen im Quai d'Orsay über Frankreichs Marschroute bezüglich der „relance européenne " 86 wurde Ende April indes deutlich, daß zumindest die Mitarbeiter des CEA eine atomare Zusammenarbeit im Rahmen der Sechsergemeinschaft als kaum sinnvoll erachteten, weil Frankreich ihrer Meinung noch von den kleinen Partnerstaaten keinen Nutzen erwarten konnte. Angesichts der restriktiven Haltung der Amerikaner gegenüber den eigenen Kooperationswünschen und der Sonderrolle Englands schien sich nur eine Verbindung mit Deutschland auszuzahlen ${ }^{87}$. Dieser Befund warf freilich ein Dilemma auf. Mit der unmittelbar bevorstehenden Ratifikation der Pariser Verträge und der Entlassung der Bundesrepublik in die Souveränität drohte der „cauchemar d'un programme atomique germano-américain dépassant le pro-

82 S. Pressekonferenz Faures, 13. 4. 1955, in: AdG 1955, S. 5114B; Gerbet, "Relance“, S. 75 f.

83 S. Goldschmidt, Rivalités, S. 207 f.

84 S. ebd., S. 209; Weilemann, Anfänge, S. 39.

85 S. IMPF, Dossier Défense nationale, Carton 1, Ailleret an Binoche, Aufzeichnung, o.D.; ebd., Aufzeichnung an Pelabon, 22. 1. 1955.

86 Vgl. Kap. IV.2.1.

87 S. AMAE, DE-CE, Bd. 611, Bl. 67-77, Interministerielle Treffen vom 22. u. 25. 4. 1955. 
gramme français" 88 . Versuchte man die Deutschen in eine nukleare Interessengemeinschaft zwischen beiden Staaten oder im Verbund der Sechs zu ziehen, bestand die Gefahr, daß sie eine prädominante Stellung einnähmen ${ }^{89}$. Als entschiedener Befürworter der deutsch-französischen Nuklearkooperation rief Sauvagnargues, der mittlerweile als Conseiller technique im Kabinett des Außenministers Pinay fungierte, seine Kollegen aus den technischen Ressorts dazu auf, der Bundesrepublik nicht den Eindruck zu vermitteln, „que la France désirerait sous prétexte de coopération, réintroduire des contrôles“. Die von Bonn im Rahmen der Pariser Verträge akzeptierte Höchstmenge spaltbaren Materials bot für ihn kein Hindernis gemeinsamer Projekte, da er sie nur als „un simple programme“ verstand. Bertrand Goldschmidt, der Directeur des relations extérieures des Commissariat à l'Energie Atomique zeigte sich insbesondere an einem zweistaatlichen Bau einer Isotopentrennanlage interessiert, wenig hingegen an einem gemeinsamen Zentrum für Angewandte Nuklearforschungen90. Als Fachmann wußte er natürlich, daß die Isotopentrennung technisch zur Produktion des Isotops Uran 235 notwendig war, das sich zur Kernspaltung eignete und im Vergleich zu natürlichem Uran 238 eine höhere Effektivität aufwies. Das Verfahren zur Urananreicherung beherrschten aber nur die USA, Großbritannien und die Sowjetunion. Da die Amerikaner lediglich begrenzte Mengen abgaben und Bemühungen um technische Hilfe der Briten an englisch-amerikanischen Abmachungen scheiterten, sahen sich die Franzosen fast notgedrungen auf die Deutschen verwiesen ${ }^{91}$.

Umgekehrt besaß aber auch die Bundesrepublik in der nun beginnenden Aufbauphase der Bundeswehr ein großes Interesse an einer Zusammenarbeit mit dem Nachbarn, mußte sie doch „am Punkt Null“ mit der Aufstellung einer 500000 Mann umfassenden vollständigen Armee beginnen ${ }^{92}$. Frankreich nutzte diese Situation aus Gründen des Prestiges wie der wirtschaftlichen Interessen aus, um nicht im harten Kampf gegen englische und amerikanische Konkurrenten das Nachsehen zu haben ${ }^{93}$. Eingeweihte deutsche Politiker beobachteten mitunter mit „Ekel [...], wie Regierungen, Industrieverbände, Einzelfirmen usw. das deutsche Rüstungsgeschäft als die große Chance sehen, aus der sich alle möglichen Vorteile erzielen lassen." 94

In diesem gigantischen Rüstungspoker ging es natürlich nicht nur um die kurzfristige Ausstattung von Kasernen und Soldaten, sondern auch um mittel- und langfristige Rüstungsprojekte. Wie eine Unterredung zwischen den Abteilungsleitern im Auswärtigen Amt und im Quai d'Orsay, von Maltzan und Wormser, vom 30.4. zeigte, wollte Bonn die militärische Kooperation im Rahmen des WEU-Rüstungsausschusses durchgeführt sehen und sprach sich gegen die Schaffung eines zweiseitigen Organs aus. Paris hingegen setzte auf Bilateralität, ohne den übrigen Partnern in der WEU den Zugang zu versperren. Besonderes Inter-

88 Guillen, La France et la négociation des traités de Rome, S. 514.

89 S. Aufzeichnung des MAE, o.D., in: DDF 1955, Bd. 1, S. 547.

90 AMAE, DE-CE, Bd. 611, Bl. 67-77, Interministerielle Treffen vom 22. u. 25. 4. 1955; s.a. Aufzeichnung des MAE, o.D., in: DDF 1955, Bd. 1, S. 546-551.

91 Vgl. Scheinman, Atomic energy, S. 176; Weilemann, Anfänge, S. $161 \mathrm{f}$.

92 Greiner, Eingliederung, S. 563.

93 S. Bericht Crouziers, o.D., Très secret, in: BDFD I, S. 671-674.

94 DLA, NL Hausenstein, 66.2562, Strauß an Hausenstein, 5. 4. 1957. 
esse signalisierte Wormser im Bereich der Luftfahrt und bei der Isotopentrennung, während von Maltzan auch die Forschung mit einbezogen zu sehen wünschte ${ }^{95}$.

Daß die Bundesregierung aber sehr wohl auch die zwischenstaatliche Perspektive verfolgte, verdeutlichten nicht zuletzt die Bemühungen des präsumtiven Chefs des Bundesverteidigungsministeriums, Theodor Blank. Nachdem er bereits im November 1954 Kontakte mit dem Staatssekretär im französischen Verteidigungsministerium, Crouzier, aufgenommen hatte ${ }^{96}$, verabredete er Mitte Mai 1955 am Rande einer Vorführung ausländischer Schulflugzeuge für die Bundeswehr mit Angehörigen der französischen Botschaft einen Besuch in Frankreich. FrançoisPoncet begrüßte diese Reisepläne ausdrücklich und empfahl Pinay, mit der Einladung nicht zu lange zu warten, weil Amerikaner und Engländer schon stark um die Gunst der Deutschen buhlten. Um die Visite zu einem echten Erfolg werden zu lassen, durfte sie sich seiner Meinung nach nicht nur darin erschöpfen, dem Gast jene Geräte zu zeigen, die er zu sehen wünsche. Sie sollte vielmehr die Basis für die zukünftige deutsch-französische Militärkooperation legen und daher Blank die Möglichkeit geben, nach seiner Rückkehr präzise Vorschläge für die gemeinsame Produktion und Ausrüstung, für technische Forschungen und Experimente zu machen ${ }^{97}$.

Das französische Verteidigungsministerium mochte dem Botschafter in seiner Planung aber nicht folgen. Koenig wollte den Besuch nicht so hoch aufhängen, ihm einen rein technischen Charakter geben und ohne großes Protokoll ablaufen lassen ${ }^{98}$. Blank wurde daher nach Absprache mit dem Quai d'Orsay und dem Matignon lediglich dazu eingeladen, einige Installationen bzw. Fabriken zu besuchen und Luftfahrtpräsentationen beizuwohnen99. Er war mit einem derartig beschränkten Programm aber nicht einverstanden, da er eine kleine Expertengruppe mitzunehmen gedachte und sich auch für Panzer und Panzerabwehrwaffen interessierte. Bei François-Poncet traf er auf offene Ohren. Noch einmal regte der Botschafter im Quai d'Orsay an, dem Besuch eine höhere Bedeutung beizumessen und ihn als Grundlage für die kommende Militärkooperation zu nutzen ${ }^{100}$.

Blanks Visite Mitte Juni erhielt dann tatsächlich einen imposanteren Rahmen, als Koenig ihm zunächst zugeben wollte. Gewiß spielte dabei auch eine Rolle, daß er wenige Tage zuvor zum Verteidigungsminister ernannt worden war und damit aus einer gestärkten Position heraus auftreten konnte. Vor allem aber, so scheint es, hatte sich das drängende Außen- gegenüber dem zögerlichen Verteidigungsministerium durchgesetzt. In Begleitung von General Speidel und verschiedenen anderen hohen Offizieren angereist, wurden ihm am 14.6. auf den Feldern von

${ }^{95}$ S. Unterredung zwischen Wormser und von Maltzan vom 30.4. 1955, Confidentiel, in: BDFD II, S. 448-456.

96 Vgl. AMAE, Europe 1944-1960, Allemagne, Bd.207, Bl. 150-155, „Coopération franco-allemande sur le plan aeronautique", 18. 11. 1955, Secret.

${ }_{97}$ S. ebd., Bd. 389, Bl. 198-200, François-Poncet an Pinay, Nr. 513/HC/AM, 13. 5. 1955, Secret; Bossuat, Armements, S. 605.

98 S. AMAE, Europe 1944-1960, Allemagne, Bd. 389, Bl. 216, Koenig an Palewski, Nr. 711/DN/ $\mathrm{CAB} / \mathrm{S}, 21.5 .1955$, Secret.

99 S. ebd., Bl. 217, Crouy-Chanel an Bonn, Tel. 2398/99, 24. 5. 1955.

100 S. ebd., Bd. 389, Bl. 218, François-Poncet an MAE, Tel. 2033/38, 25. 5. 1955. 
Frileuse und Satory zunächst Geräte vorgeführt. Sodann empfing ihn Palewski zum Déjeuner. Anschließend traf er sich mit Koenig. General Guillaume, Chef d'Etat-Major Général des Forces Armées, und die drei Chefs der Teilstreitkräfte gaben ihm zu Ehren ein Essen, wobei Guillaume nicht zu betonen vergaß, daß die drei Generalstabschefs nie zuvor einen deutschen Verteidigungsminister gemeinsam empfangen hätten. Das für den folgenden Tag vorgesehene Programm mußte kurzfristig abgesagt werden, weil Blank nach Bonn zurückeilte, um im Bundestag seinen Haushalt zu verteidigen. Er kehrte aber direkt wieder nach Paris zurück, besuchte am 16.6. die Luftfahrtschau in Le Bourget und traf sich mit Crouzier zum Déjeuner. Folgt man den französischen Berichten, zeigte er sich von der Leistungsfähigkeit der französischen Rüstungsgüter sehr angetan, wobei ihn insbesondere Fernlenkwaffen und der Panzer AMX sowie die neuesten Modelle der Luftwaffe von den Typen „Vautour“, „Trident“ und „Mystère“ beeindruckten. Persönlich lebhaftes Interesse äußerte er an einer Rüstungszusammenarbeit in der Artillerie, beim Panzerbau und in der Luftwaffe von der Logistik über die Forschung bis zur Produktion, sofern sie sich völlig gleichberechtigt und ohne Hintergedanken abspiele. Da der Bundesrepublik aktuell von vielen Seiten Angebote zugingen, bat er um absolute Geheimhaltung 101 .

Bonns Wunsch nach einer "coopération étroite et confiante“ wirkte so überzeugend, daß er - angesichts der ursprünglichen Reserven auf Pariser Seite durchaus überraschend - lebhaft erwidert wurde. Die ersten Schritte schienen recht ermutigend. Nun mußte der gemeinsame "désir sincère" in greifbare Projekte und präzise Abmachungen umgesetzt werden ${ }^{102}$. Allerdings war man sich sehr wohl bewußt, daß die Entscheidung über das Ausmaß der Kooperation von zahlreichen Unwägbarkeiten abhing wie der Organisation der Rüstungsproduktion in Deutschland, den Konkurrenzangeboten der Amerikaner oder Engländer oder den Rüstungslieferungen der USA ${ }^{103}$. Blank hatte als erste konkrete Maßnahmen Besprechungen zwischen Experten beider Seiten und Kontakte zwischen militärischen Führungsebenen zur Definierung der quantitativen und qualitativen Erfordernisse vereinbart. In der Rue Saint-Dominique hoffte man nun, die deutschen Techniker in den französischen Laboratorien und Forschungsstellen halten zu können. Man strebte Abmachungen über gemeinsame oder komplementäre Rüstungsproduktionen an und zielte auf die Errichtung gemeinsamer Depots der deutschen bzw. französischen Truppen in Deutschland 104.

Wenige Wochen nach dem Besuch des Verteidigungsministers setzte die Bundesregierung ein leuchtendes Signal. Sie kaufte französische „Hotchkiss“-Panzer zu Prüfungszwecken, gab den etwa einhundert deutschen Mitarbeitern im ballistischen Forschungszentrum Saint-Louis im Oberelsaß die Anweisung, bis auf weiteres vor Ort zu bleiben, und führte erste Gespräche über den Kauf von Lizenzen für das unter maßgeblicher Mitwirkung deutscher Ingenieure gebaute Düsen-

101 S. ebd., Bd. 389, Bl. 251f., Unterredung zwischen Blank und Palewski vom 14.6. 1955; ebd., B1. 273-275, Aufzeichnung Leroy-Beaulieu, 18.6. 1955, Très secret; Bossuat, Armements, S. $605 \mathrm{f}$. 102 Aufzeichnung Legendre, 24. 6. 1955, in: BDFD I, S. 665 f., hier S. 666.

${ }_{103}$ S. AMAE, Europe 1944-1960, Allemagne, Bd. 389, Bl. 273-275, Aufzeichnung Leroy-Beaulieu, 18. 6. 1955, Très secret.

104 S. Aufzeichnung Legendre, 24. 6. 1955, in: BDFD I, S. 665. 
triebwerk ATAR ${ }^{105}$. Unzufriedenheit im Verteidigungsministerium mit der Technik des ursprünglich zur Anschaffung vorgesehenen britischen Jagdflugzeuges „Hawker Hunter“ führte außerdem zu der Überlegung, die für die Luftwaffe vorgesehenen 300 Maschinen zumindest teilweise durch französische „Mystère IV“ zu ersetzen. Da eine entsprechende Entscheidung durch amerikanische Stellen genehmigt werden mußte, wandten sich die zuständigen deutschen Offiziere im Verteidigungsministerium an die französische Botschaft und baten um Unterstützung. François-Poncet trat einmal mehr als Sprachrohr deutscher Rüstungsambitionen auf, erinnerte Pinay an das beträchtliche Interesse, das Blank anläßlich seines Besuches der „Mystère“ entgegengebracht hatte, und empfahl, diese wichtige und delikate Frage mit der ihr gebührenden Aufmerksamkeit zu verfolgen ${ }^{106}$.

Im Quai d'Orsay rannte er damit offene Türen ein. Im Gegensatz zum Verteidigungsministerium gingen die Ambitionen des Außenministeriums nämlich inzwischen weit über die bisherigen Projekte hinaus. Man begnügte sich nicht mehr mit dem Austausch von Ressourcen, sondern strebte echte Gemeinschaftsprojekte an, um eine langfristige, durch vertraglich Abmachungen abgesicherte $\mathrm{Zu}$ sammenarbeit zu gewährleisten, die zu einem späteren Zeitpunkt auch in den gröBeren Rahmen des Rüstungsausschusses der WEU gestellt werden konnte ${ }^{107}$. Zweifellos reflektierten diese Planungen auch die Unzufriedenheit der deutschen Wirtschaft mit den bisherigen Ergebnissen in der Luftfahrtkooperation. Da trotz monatelanger Gespräche keinerlei definitive Resultate erzielt worden waren, gewannen die Unternehmer den Eindruck, Frankreich gehe es vornehmlich darum, „das Wiedererstehen einer unabhängigen deutschen Luftfahrtindustrie zu verhindern“. Die angebotenen Lizenzen zum Nachbau französischer Konstruktionen galten ihnen als inakzeptabel, zumal sie sich nicht langfristig verpflichten und die Freiheit zum Abschluß auch mit angelsächsischen Firmen bewahren wollten ${ }^{108}$.

Ende Juli lagen dem Matignon auf der Basis innerministerieller Absprachen allgemeine Richtlinien für die deutsch-französische Rüstungszusammenarbeit vor, „pour servir de base à une entente plus large dans le cadre de l'Union de l'Europe Occidentale“. Darin sprach sich die Regierung für die Fortsetzung der deutschen Beteiligung an den Pariser Forschungslaboratorien aus, um die Funktionsfähigkeit dieser Institutionen zu gewährleisten. Sie sah außerdem sowohl reine Kaufals auch gemeinsame oder komplementäre Produktionsprogramme vor, stellte $\mathrm{Fa}$ brikationslizenzen in Aussicht, sicherte gegenseitige Hilfe bei der Technikerausbildung zu und plante die Vorbereitung gemeinsamer Beschaffungsprogramme ${ }^{109}$.

105 S. AMAE, Europe 1944-1960, Allemagne, Bd. 208, Bl. 34-37, Aufzeichnung der Unterabt. Zentraleuropa im MAE, 30.6. 1955 (Konzept ML). Zu dem von deutschen Technikern nach dem Zweiten Weltkrieg bei Basel auf französischem Territorium aufgebauten Forschungszentrum St. Louis vgl. Kocs, Autonomy, S. 80 f. u. Albrecht, Rüstungsfragen, S. 153 f.; zum ATAR-Triebwerk vgl. ebd., S. 143-146; Bossuat, Armements, S. 603.

106 S. AMAE, Europe 1944-1960, Allemagne, Bd. 208, Bl. 32 f., François-Poncet an Pinay, Nr. 526/ EU, 27. 6. 1955, Très secret.

107 S. ebd., Bl. 34-37, Aufzeichnung der Unterabt. Zentraleuropa im MAE, 30. 6. 1955 (Konzept ML).

108 Von Maltzan an AA, 307 a 78 W 1210/55, 23. 7. 1955, in: BDFD II, S. 1028 f., hier S. 1028.

109 „Directives Générales relatives à la Coopération franco-allemande en matière d'armement“, o.D., Très secret, in: BDFD I, S. 666 f., hier S. 666. Das undatierte und von Regierungschef Faure gebilligte Dokument wurde am 29.7. 1955 an die Ressortminister geschickt (AMAE, Europe 1944- 
Am 1. 9. empfahl François-Poncet Adenauer als Einstieg in die beiderseitige Rüstungskooperation die Bestellung von einigen „Morane“-Flugzeugen, und der Kanzler versprach, sich beim Verteidigungsminister dafür einzusetzen ${ }^{110}$. Zwei Wochen später legte die Bundesregierung in Paris eine ansehnliche Wunschliste über einen Gesamtwert von sechzig Milliarden Francs vor. Sie umfaßte unter anderem: Bestellung von fünfzig Schulflugzeugen vom Typ „Morane 733“; Bitte um Produktionslizenzen für die Transportmaschine „Nord 2501“ sowie für das Schulflugzeug „Potez-Air Fouga CM-170“, dessen Produktion von Messerschmidt durchgeführt werden sollte. Interesse meldete Bonn außerdem für das Kampfflugzeug „Mystère“ und diverse Rüstungsgüter für Heer und Marine an. Im Gegenzug dachte Frankreich an die gemeinsame Benutzung logistischer Anlagen auf deutschem Boden, an die Überlassung einiger Praktikantenplätze an französischen Technikschulen und an die Kooperation in der Forschung, insbesondere zwecks Fortsetzung der Arbeitsverträge der deutschen Techniker im Forschungszentrum Saint-Louis 111 .

$\mathrm{Zu}$ ersten, noch provisorische Kontrakten kam es im Oktober zwischen der "Société Nationale de Construction Aéronautique de Nord“ (SNCAN) und der Firma Siebel über die Fabrikation des Transportflugzeugs "Nord 2501“ sowie zwischen Fouga und Messerschmidt über den Bau des „Fouga Magister“. Ende des Monats einigte sich das Bundesverteidigungsministerium mit dem Ministerium für die Nationale Verteidigung und die Armeen im Prinzip darauf, SaintLouis in eine gemeinsame Forschungsanstalt umzuwandeln. Des weiteren beschlossen sie, das Laboratorium für Ballistik und Aerodynamik in Vernon und das "Centre d'essais de réacteurs de Melun-Villaroche“ der "Société Nationale d'Etude et de Construction de Moteurs d'Avions" (SNECMA), dem führenden Staatsbetrieb für Flugmotoren mit hochrangigen deutschen Mitarbeitern, sowohl deutschen als auch gemeinsamen Projekten zu öfnen. Blank setzte sich dabei offen über die Befugnisse des Bundeswirtschaftsministeriums hinweg und stellte sich auf erhebliche Kompetenzschwierigkeiten ein ${ }^{112}$.

Am 7. 11. trafen sich Vertreter der beteiligten französischen Ministerien im Secrétariat Général Permanent de la Défense Nationale, um die weiteren Maßnahmen, die Form der Rüstungskooperation und die zu ergreifenden Methoden für die bevorstehenden Verhandlungen mit Bonn festzusetzen. Die Runde richtete eine Arbeitsgruppe ein, um die juristischen Probleme der geplanten Abkommen zu studieren, und beauftragte das Außenministerium mit einer diplomatischen Démarche bei der Bundesregierung, damit der Streit zwischen Erhard und Blank nicht zur Belastung würde'113.

Wenige Tage nach dieser Sitzung sahen sich Bonn und Paris heftigen Attacken aus Washington ausgesetzt. Amerikanische Diplomaten machten keinen Hehl daraus, daß sie die deutsch-französischen Arrangements über die Ausrüstung der

1960, Allemagne, Bd. 206, Bl. 258f., Palewski an Pinay, Koenig, Pflimlin, Morice, Crouzier, Gilbert-Jules, Nr. 2136/DN/EG, 29.7. 1955, Très secret).

110 S, ebd., Bd. 390, Bl. 50, François-Poncet an MAE, Tel. 3459, 2. 9. 1955.

111 S. ebd., Bd. 207, Bl. 108 f., „Projets de coopération, dont la discussion a été amorcée“, 21. 10. 1955,

Secret; zum Fouga Magister vgl. Albrecht, Rüstungsfragen, S. $166 \mathrm{f}$.

112 S. Interministerielle Sitzung vom 7. 11. 1955, 15. 11. 1955, Secret, in: BDFD I, S. 667-669.

113 S. ebd., S. 669-671; zur SNECMA vgl. Albrecht, Rüstungsfragen, S. 144. 
Luftwaffe annulliert zu sehen wünschten und auf die Ausrüstung mit amerikanischem Material beharrten. Erhard machte sich diese Kritik sofort zu eigen und sprach sich "au nom d'une doctrine de libéralisme“ gegen die französischen Lieferungen aus. Aus Gründen des Prestiges wie des Prinzips mochten sich weder die französischen Diplomaten in Bonn noch die leitenden Beamten im Quai d'Orsay diesen Pressionen beugen. Und sie legten Außenminister Pinay dringend ans Herz, dem Kanzler gegenüber vor Augen zu führen, daß man die Grundlage der europäischen Kooperation aufs Spiel setzte, wenn man dem Druck amerikanischer Industrieller nachgäbe ${ }^{114}$.

Pinays Démarche am 13. 11. in Rhöndorf zeigte schon nach wenigen Tagen eine erste Wirkung. Das Bundesverteidigungsministerium erklärte sich gegenüber der

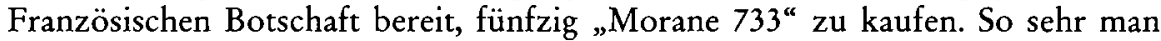
sich im Dreesen auch über diese Zusage freute, so war man angesichts der Konkurrenz aus Übersee doch weit davon entfernt, beruhigt zu sein, zumal die Amerikaner sich nun anschickten, die „Mystère“ zu verdrängen. Der sich verschärfende Wettkampf war keineswegs gewonnen, und es bedurfte weiterer Einwirkungen auf die Bundesregierung, damit sie ihre Bestellversprechungen nicht wieder zurücknahm ${ }^{115}$. Einfluß galt es für Frankreich aber auch auf die eigene Industrie zu nehmen, um sie davon zu überzeugen, daß es im internationalen Wettstreit um deutsche Rüstungsaufträge nicht ausreichte, mit den Deutschen bloße „accord[s] de licence" abzuschließen ${ }^{16}$. Dieser Wettstreit war es auch, der die Regierung in Paris in diesen Tagen dazu veranlaßte, der Bitte des neuen deutschen Atomministers Strauß um einen Informationsbesuch in Frankreich stattzugeben und ihm Mitte November bei einem zweitägigen Aufenthalt herausragende Gesprächspartner zur Verfügung zu stellen, die über Aufbau und Arbeit des Commissariat à l'Energie Atomique und das Centre d'Etudes Nucléaires in Saclay bei Versailles informierten ${ }^{117}$.

Die bei den Verhandlungen über die Europäische Atomgemeinschaft auftretenden Schwierigkeiten trugen offensichtlich mit dazu bei, die bilaterale Kooperation zu festigen, obwohl es nach der Aufnahme der Regierungsgespräche zunächst den Anschein hatte, als verlagerte sich insbesondere das Problem der Isotopentrennanlage von der bilateralen auf die europäische Ebene. Da Belgien sein Uranerz aus Belgisch-Kongo den USA zu günstigeren Konditionen lieferte als Frankreich, sahen die Franzosen im EURATOM-Projekt nicht zuletzt eine Möglichkeit, das US-Monopol zur Herstellung angereicherten Urans zu brechen. Als eine der Hauptaufgaben der neuen Gemeinschaft betrachteten sie daher den Bau einer Isotopentrennanlage ${ }^{118}$. Implizit ergab sich für sie dabei aber das Problem, inwieweit

114 S. AMAE, Europe 1944-1960, Allemagne, Bd. 207, Bl. 122 f., Generaldirektion Politik im MAE an Pinay, Aufzeichnung, 12.11. 1955; de Margerie an dens., Tel. 4330/32, 10.11. 1955, Réservé, in: DDF 1955, Bd. 2, S. $791 \mathrm{f}$.

115 S. AMAE, Europe 1944-1960, Allemagne, Bd. 207, Bl. 144, ders. an dass., Tel. 4439/40, 17. 11. 1955, Réservé.

116 Ebd., Bd. 207, Bl. 150-155, „Coopération franco-allemande sur le plan aéronautique*, 18.11. 1955, Secret.

117 S. ebd., Bd. 390, Bl. 156f., de Margerie an Pinay, Nr. 1425, 22. 10. 1955; ebd., Bl. 200f., Bericht über den Aufenthalt von Strauß in Paris vom 15./16. 11. 1955, Nr. 55-1618, 23. 11. 1955.

118 Vgl. Eckert, Kernenergie, S. 319; Guillen, La France et la négociation du traité d'Euratom, S. 396398 u. 404-407; Weilemann, Anfänge, S. 37 f.; Kap. IV.2.1. 
sie sich bei der zivilen Anwendung der Kernenergie europäisch binden konnten, ohne die eigenen militärischen Atomambitionen zu fesseln. Jene Funktionäre, die wie Goldschmidt oder Guillaumat, der Administrateur général des Commissariat à l'Energie Atomique, den Schwerpunkt auf die militärische Nutzung setzten, sprachen sich gegen jegliche supranationale Organisation im Nuklearbereich aus ${ }^{119}$. Auch im Quai d'Orsay vertrat man im Herbst 1955 die Auffassung, man solle zunächst die Zusammenarbeit mit den Deutschen auf bilateraler Ebene suchen und den zivilen bzw. militärischen Flugzeugbau sowie die Konstruktion der Isotopentrennanlage forcieren. In dem Maße, wie diese Projekte das Vertrauen in die Wirksamkeit der europäischen Idee wiederbelebten, glaubten die Beamten auch die größeren Pläne wie die Atomgemeinschaft umsetzen zu können ${ }^{120}$. Vor diesem Hintergrund lud Verteidigungsminister Blank den französischen Staatssekretär Crouzier am 23. 11. in der Absicht zu einem Besuch nach Bonn ein, die beiderseitige Kooperation zu präzisieren ${ }^{121}$. In einer zur Vorbereitung dieses Treffens anberaumten Gesprächsrunde verständigten sich Beamte der zuständigen französischen Ministerien am 1. 12. darauf, die Umformung von Saint-Louis in eine deutsch-französische Organisation und den Vertrag zwischen SNCAN und Siebel zum Bau des „Nord 2501“ zu billigen. Demgegenüber sollte der Vertrag zwischen Fouga und Messerschmidt neugefaßt werden, weil der Text zu stark auf sofortige kommerzielle Interessen ausgerichtet schien, da er nur die Überlassung einer Lizenz enthielt und damit nicht den Regierungsdirektiven entsprach ${ }^{122}$.

Mit dieser Marschroute im Gepäck reiste Crouzier eine Woche später an den Rhein, wo er zunächst von seinem deutschen Kollegen Josef Rust empfangen wurde. Beide waren sich rasch einig, daß die Politik der bilateralen Verständigung und die beiderseitige Sicherheitspartnerschaft in der NATO den beiden Nachbarn gar keine andere Wahl ließ, als dieselbe Rüstungspolitik zu betreiben. Blank bestätigte die Ausführungen seines Staatssekretärs, schränkte sie aber insofern ein wenig ein, als er auf die Notwendigkeit hinwies, nicht mit der Freihandelspolitik des Wirtschaftsministeriums in Konflikt zu geraten. $\mathrm{Zu}$ den konkreten Projekten überleitend, billigte er das Projekt der Umwandlung des Laboratoriums SaintLouis in ein deutsch-französisches Forschungsinstitut ebenso wie die Verträge zwischen Siebel und SNCAN bzw. Fouga und Messerschmidt. Crouzier nutzte die sehr entspannte Gesprächsatmosphäre, um für weitere französische Rüstungsgüter zu werben. So strich er die Vorteile einer Ausrüstung der Bundesluftwaffe mit den von Frankreich benutzten leichten Abfangägern und taktischen Flugzeugen heraus, was bei Blank auf eine günstige Reaktion stieß ${ }^{123}$. Nachdem er in wesentlich schwierigeren Gesprächen mit Staatssekretär Westrick vom Bundeswirtschaftsministerium dessen Billigung zu den drei Kooperationsprojekten er-

119 S. Guillen, La France et la négociation du traité d'Euratom, S. 395; Goldschmidt, Rivalités, S. 209213.

120 S. Aufzeichnung der Unterabt. Zentraleuropa im MAE, 21. 11. 1955, Confidentiel, in: DDF 1955, Bd. 2, S. 840 .

121 S. AMAE, Europe 1944-1960, Allemagne, Bd. 390, Bl. 213, Blank an Crouzier, 23. 11. 1955 (Übersetzung).

122 S. ebd., Bd. 207, Bl. 170-180, Interministerielle Sitzung vom 1. 12. 1955, 7. 12. 1955, Secret.

123 S. Bericht Crouziers, o.D., Très secret, in: BDFD I, S. 671-674. 
halten hatte ${ }^{124}$, kehrte Crouzier mit der Gewißheit nach Paris zurück, daß die Zeit für beiderseitige Rüstungsgeschäfte wegen der großen Schwierigkeiten beim Aufbau der Bundeswehr äußerst günstig sei: „Notre pays peut et doit jouer un rôle déterminant dans cette phase critique de début du réarmement allemand. En dehors des questions de prestige à maintenir, il y a que nous pouvons assurer à notre industrie d'armement une place de choix si nous savons persévérer dans l'action très rigoureusement coordonné que nous menons dans le domaine de la coopération militaire Franco-Allemande depuis le mois de juin dernier." ${ }^{125}$ Der Staatssekretär erwartete in Kürze neue Gespräche, da die Bundesrepublik sehr bald wichtige Entscheidungen über die Aufrüstung ihrer Armee zu treffen hatte ${ }^{126}$. Freilich ging es ihm nicht nur um das Geschäft. Frankreich machte sich auch Hoffnungen darauf, das Know how der Deutschen für die Modernisierung der eigenen Streitkräfte zu nutzen. 